\title{
Photopatterning of PMMA Films with Gold Nanoparticles: Diffusion of $\mathrm{AuCl}_{4}{ }^{-}$Ions
}

\author{
Eda Yilmaz, Gulay Ertas, Erman Bengu, and Sefik Suzer* \\ Department of Chemistry, Bilkent University, 06800 Ankara, Turkey \\ Received: July 18, 2010; Revised Manuscript Received: September 29, 2010
}

\begin{abstract}
Photopatterning of poly(methyl methacrylate) (PMMA) films is performed by UV irradiation of the polymer films containing uniformly distributed $\mathrm{AuCl}_{4}{ }^{-}$ions. The process reduces the gold ions and leads to production of Au nanoparticles in the irradiated regions at room temperature (RT). Resulting films are investigated with scanning electron microscopy, which revealed, in addition to regions with gold nanoparticles, the presence of "ion-depleted regions". These regions are formed at RT and within the rigid polymer matrix by diffusion of gold ions toward the irradiated regions, ending up with no or very little gold moieties, which are important for prevention of delayed processes for postgeneration of unwanted features, if and when such materials are utilized for device production. Further investigations performed by fluorescence and Raman measurements and XPS mapping give additional evidence supporting the existence of such regions. Similar regions are also observed within the poly(vinyl alcohol) (PVAL) films. The ion-depleted regions are about $10 \mu \mathrm{m}$ wide, which is a significant length for the metal ions to travel through a rigid matrix like PMMA (or PVAL) at room temperature and raises important questions as to the diffusion mechanism(s) of the metal ions and to the nature of the driving force(s).
\end{abstract}

\section{Introduction}

Polymer-nanoparticles are technologically important composite systems, gaining increasing interest from many different fields of science and technology in the past decade. ${ }^{1-4}$ Such composites are seen as fundamental components for the next generation of light and flexible electronic devices. Therefore, producing such materials in a controlled way is of upmost importance and still presents several challenges.

Production of polymer-nanoparticle systems can be achieved by many different approaches, such as integration of nanoparticles (NPs) in the polymer matrix after their synthesis. With this approach, a good and homogeneous composite can be produced, but controlling spatial distribution of the nanoparticles is not possible. ${ }^{5}$ On the other hand, synthesizing nanoparticles inside the polymer matrix starting with a metal precursor enables control of the distribution of nanoparticles inside the polymer matrices. For example, using polymer blends as the synthesis medium one can create patterns with immiscible polymers and selectively adsorb metal precursors and synthesize metal nanoparticles in one polymer and leave the other polymer free of nanoparticles. ${ }^{6,7}$ Another way to synthesize nanoparticles in a polymer film is to start with a well-dispersed metal ion precursor and use energetic light to induce reduction by providing energy to the polymer matrix and creating electron donor centers. Once the metal ions are photoreduced, the resulting gold atoms can diffuse out relatively easily within the polymer matrix toward nucleation centers guided by the large driving force due to the cohesive energy involved. ${ }^{8-13}$ This method also enables photopatterning applications since one can synthesize nanoparticles at places where the light impinges.

Photopatterning also brings out issues concerning the fate of the metal ions or the precursors left out in the dark regions, since during the life span of these materials, further exposure to energetic photons is unavoidable. Although, in comparison

* To whom correspondence should be addressed. E-mail: suzer@ fen.bilkent.edu.tr. Phone: +90-312-2901476. Fax: +90-312-2664068. to the initial UV exposure, postpreparation exposures are likely to be in much smaller doses, cumulative effects cannot be ignored at all. In our particular case of the UV patterning, one would like to consume all of the $\mathrm{Au}^{3+}$ ions even in the unexposed regions, or somehow make them move to the illuminated ones, all during the initial exposure period, to ultimately end up with ion-depleted regions of significant dimensions. There are several articles related to metal atom movement/diffusion and nanoparticle growth within polymers, mostly above the glass transition temperature of the polymeric material, ${ }^{14-16}$ but, to the best of our knowledge, there is no report dealing with diffusion of ions or precursors. This knowledge is pertinent for utilization of these composites with respect to important issues related to their durability, aging effects, etc.

In this study, we present a thorough experimental investigation of photopatterning of poly(methyl methacrylate) matrix with gold nanoparticles using UV irradiation. The photopatterning is performed by a simple masking technique, and the patterns are faithfully replicated on the polymer films as revealed by their optical as well as SEM images. In addition, we have observed that at the very borders between the irradiated and masked regions, there exists strips where gold ions have supposedly diffused out toward the irradiated ones, forming iondepleted regions. This phenomenon was first observed in the SEM images and then was substantiated by the additional evidence we have obtained from fluorescence images, Raman, and XPS measurements. Similar observations were also seen in poly(vinyl alcohol) composites. These ion-depleted regions are about $10 \mu \mathrm{m}$ wide, and this large length itself is remarkable for gold ions to diffuse within a solid matrix at room temperature. These observations may present valuable insight for diffusion mechanism(s) of metal ions inside polymer matrices and for the driving forces behind such large displacement(s).

\section{Experimental Section}

Poly(methyl methacrylate) (PMMA), poly(vinyl alcohol) (PVAL), Rhodamine 6G, and tetrachloroauric acid, $\mathrm{HAuCl}_{4}$, 


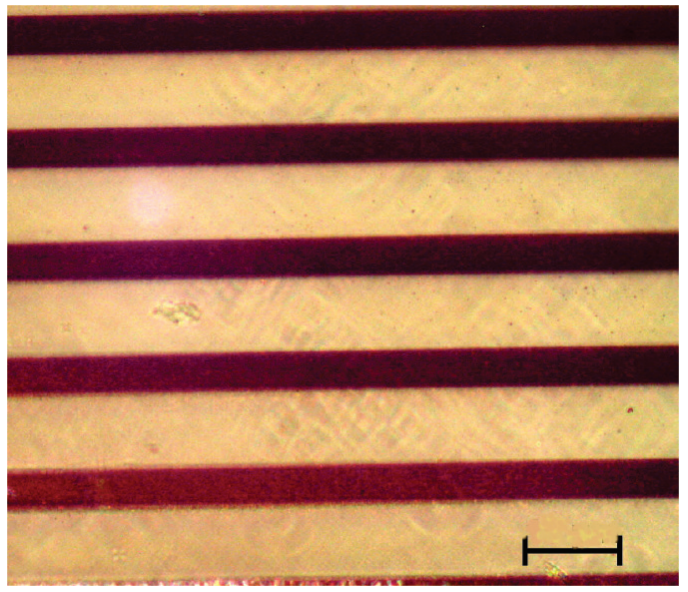

Figure 1. Optical microscope image of photopatterned PMMA film containing dispersed gold ions. Au nanuparticles are formed in the UVexposed regions (dark red). The scale bar corresponds to $10 \mu \mathrm{m}$.

were purchased from Aldrich and used without further purification. Solutions containing various concentrations $\left(1.0 \times 10^{-4}\right.$ to $1.0 \times 10^{-3} \mathrm{M}$ ) of $\mathrm{HAuCl}_{4}$ and $1 \%(\mathrm{w} / \mathrm{v})$ PMMA (or PVAL) in acetone were used to prepare the films. The thin films ( 30-60 nm thick) used for SEM measurements were prepared by spin coating on $\mathrm{Si}$ wafers, which were treated with $\mathrm{HF}$ to remove the native oxide layer prior to use. Thicker films $(\sim 10-40 \mu \mathrm{m})$ were also prepared for optical investigation, by casting from more concentrated polymer solutions, via room temperature evaporation of the solvent in a saturated acetone atmosphere. The resulting films were exposed to $254 \mathrm{~nm} \mathrm{UV}$ light from a low pressure $10 \mathrm{~W} \mathrm{Hg}$ lamp, for varying amounts of time from $30 \mathrm{~min}$ to $24 \mathrm{~h}$, to implement the complete photoreduction of gold ions and ensure formation of gold nanoparticles.

SEM images were recorded by a Carl-Zeiss EVO series scanning electron microscope. Raman measurements were performed using a Jobin-Yvon LABRAM Raman spectrometer equipped with $632 \mathrm{~nm}$ red laser, and XPS measurements were carried out using a Thermo Fischer K-Alpha spectrometer.

For fluorescence studies, a $1.0 \times 10^{-4} \mathrm{M}$ solution of Rhodamine 6G (R6G) was prepared in acetone and added to the polymer-gold salt mixture $(0.2 \mathrm{~mL}$ of R6G sol. $+0.8 \mathrm{~mL}$ of $5 \times 10^{-3} \mathrm{M} \mathrm{HAuCl}_{4}+1 \mathrm{~mL}$ of $1 \%$ PMMA). The films were prepared using three routes: route $\mathrm{A}$, films in the presence of R6G, gold salt, and polymer; route B, R6G and polymer; route $\mathrm{C}$, polymer only. Fluorescence images were taken with a Zeiss Axio Imager A1 fluorescence microscope.

\section{Results and Discussion}

As mentioned above, an advantage of synthesizing gold nanoparticles within polymer films by UV irradiation is the ease of photopatterning. By using masks that are impermeable to UV, patterns of any desired shape can be imprinted on polymers. The resulting patterns consist of two distinct regions; regions containing gold nanoparticles are the irradiated regions, and regions still containing gold ions are the masked ones. The size of photopatterns we have used can vary between $1 \mu \mathrm{m}$ to millimeters, and the thickness of the films can also be varied. In Figure 1, a light microscope image of a micrometer sized pattern on a thick film is shown. The large optical contrast between regions containing nanoparticles (dark red) and virgin regions (yellow) for such thick films of about 5-20 $\mu \mathrm{m}$ thickness is satisfactory for many potential applications.

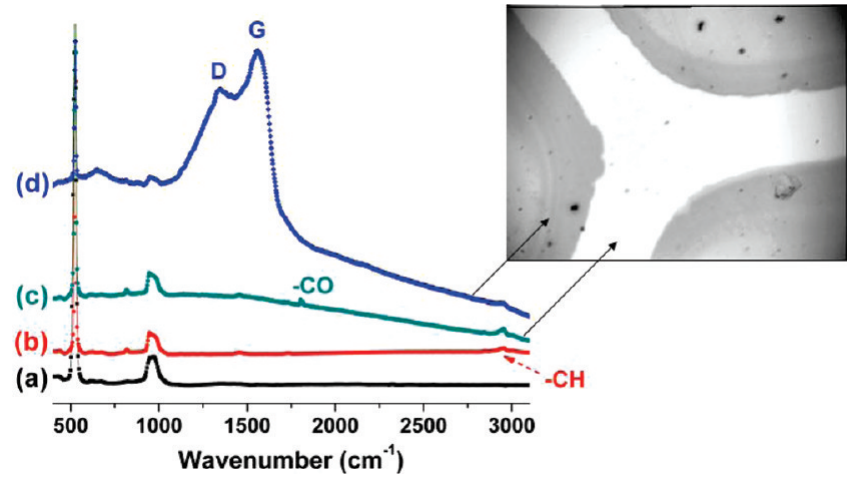

Figure 2. Raman Spectra recorded from different regions of the photopatterned film on a silicon wafer substrate: (a) substrate only and (b) after deposition of the film; after exposure to UV irradiation, (c) masked region and (d) exposed region. $-\mathrm{CH}$ and $-\mathrm{CO}$ refer to the $-\mathrm{C}-\mathrm{H}$ and $-\mathrm{C}=\mathrm{O}$ vibrational bands, respectively, and $\mathrm{D}$ and $\mathrm{G}$ refer to different $\mathrm{C}-\mathrm{C}$ stretching bands (see text).

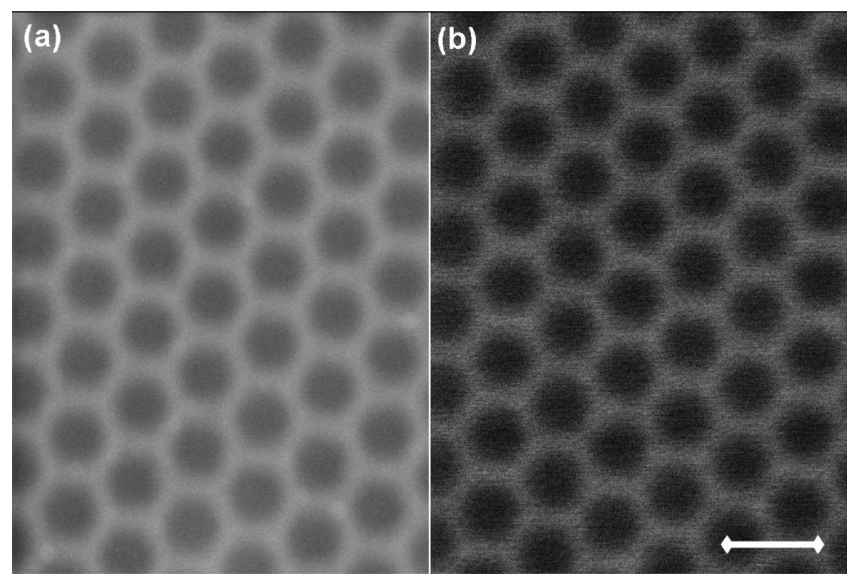

Figure 3. Secondary electron (left) and backscattered electron (right) images of a photopatterned PMMA-Au film. Metallized regions appear brighter in the images. The scale bar corresponds to $10 \mu \mathrm{m}$.

As one simple example for the application, we have recorded the Raman spectra of such patterned films from the regions which were irradiated and masked. As shown in Figure 2, the spectrum of the film before UV exposure does not yield a significant Raman signal, but after exposure, the irradiated regions give large SERS signals corresponding to the wellknown $\mathrm{D}$ and $\mathrm{G}$ bands of an organic matrix surrounding the gold nanuparticles, where the gold nanuparticles interact strongly with the red laser causing photodamage and creating graphitelike $(\mathrm{G})$ and disordered-carbon (D) moieties. ${ }^{17}$ The masked regions do not yield any Raman signal similar to the virgin film.

For very thin films with submicrometer thickness and small features, the scanning electron microscope (SEM) was used to investigate the patterns. In the SEM images, the regions containing gold nanoparticles appear brighter due to the high secondary electron yield of the gold nanoparticles as shown in Figure 3, where the contrast between the irradiated and masked regions can be seen in both the secondary electron detector (SED) and the backscattered electron detector (BSED) images. The BSED image is better due to its high detection efficiency toward heavier atoms, which is especially useful for highlighting the regions rich with gold atoms.

A crucial property of the photopatterns produced with this method is their durability. Even after the mask is removed and the films are irradiated for another $24 \mathrm{~h}$, the patterned features are still intact with no significant smearing out in the images. 


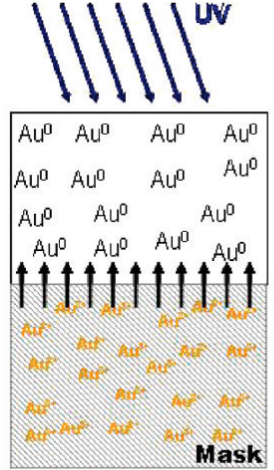

(a)

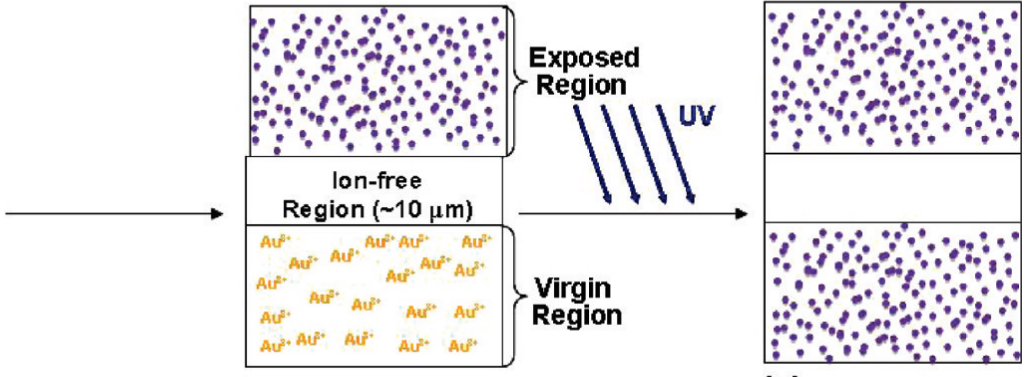

(b)

(c)

Figure 4. Schematic representation of the formation of ion-depleted regions: (a) represents the photopatterning by UV exposure of the film with the mask, (b) the film after first exposure, and (c) the film after removing the mask and applying a second exposure.

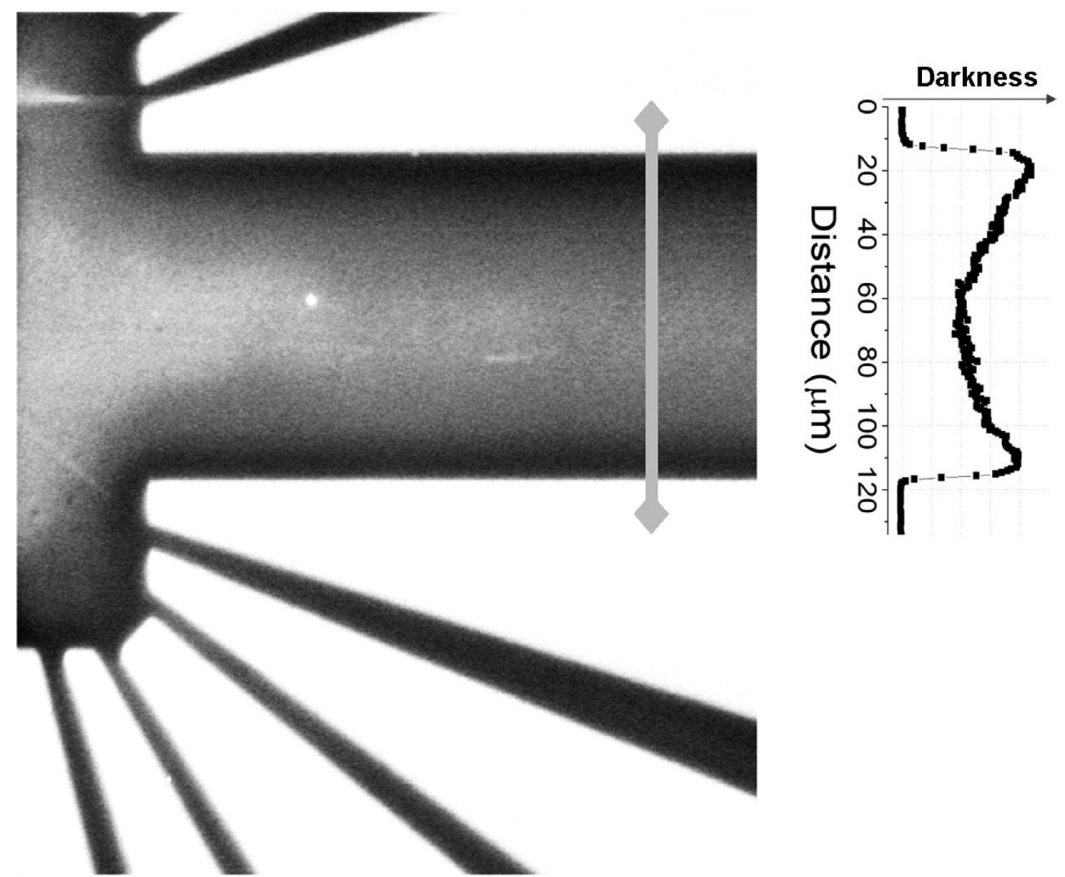

Figure 5. SEM image of the photopatterned Au-PMMA film after a second UV treatment. Inset depicts the darkness profile along the line drawn.

In addition and surprisingly, there were no significant changes in the unexposed dark regions either. This finding is very surprising and raises an important question; "Why would the further UV exposure not enable further reduction of the gold ions and formation of gold nanoparticles in the formerly dark regions?", which can only be explained by postulating that the gold ions had diffused out of these dark regions during the first patterning step.

Closer investigation of such films with SEM has revealed the existence of ion-depleted regions containing very little or no gold, at the border between irradiated and masked regions, which is supportive evidence of our postulate. The process is depicted schematically in Figure 4. These regions cannot produce gold nanoparticles even if they are irradiated for a second time after the mask is removed, and as a result, the small $(<10 \mu \mathrm{m})$ patterns are durable. Figure 5 shows another SEM image of a larger pattern and the darkness profile of such a film irradiated for the second time after the mask is removed. It is seen that in the middle region under the mask, gold nanoparticles are produced after the second irradiation, but the borders in between the irradiated and the masked regions stay dark without gold nanoparticles. The ion-depleted region observed in this image and in the others has about $10 \mu \mathrm{m}$ width. This is quite a large distance for the gold ions to travel through a solid matrix like PMMA (or PVAL), which also makes this observation interesting and sets a an important practical upper limit of $10 \mu \mathrm{m}$ for the sizes of durable photolithographical features.

As another experimental approach to understand the nature of the photopatterned films and to support our postulate for the formation of ion-depleted regions at the border regions, we have incorporated the Rhodamine 6G (R6G) into our films as an optical fluorescence contrast agent. There are several reports stating that gold ions quench the fluorescence of Rhodamine $\mathrm{B}$, whereas gold atoms do not. ${ }^{18}$ On the other hand, there are also reports that gold nanoparticles do quench the fluorescence of Rhodamine 6G. ${ }^{19}$ Whichever turns out to be true, we expect that there must exist a difference between their quenching abilities for us to use the fluorescence quenching process as a probe to distinguish between the presence of gold ions and gold nanoparticles, and/or the absence of both. We also think that there should not be too large a difference between the quenching mechanisms of Rhodamine B and Rhodamine 6GB that we used throughout our work. In the measurements we have carried out 


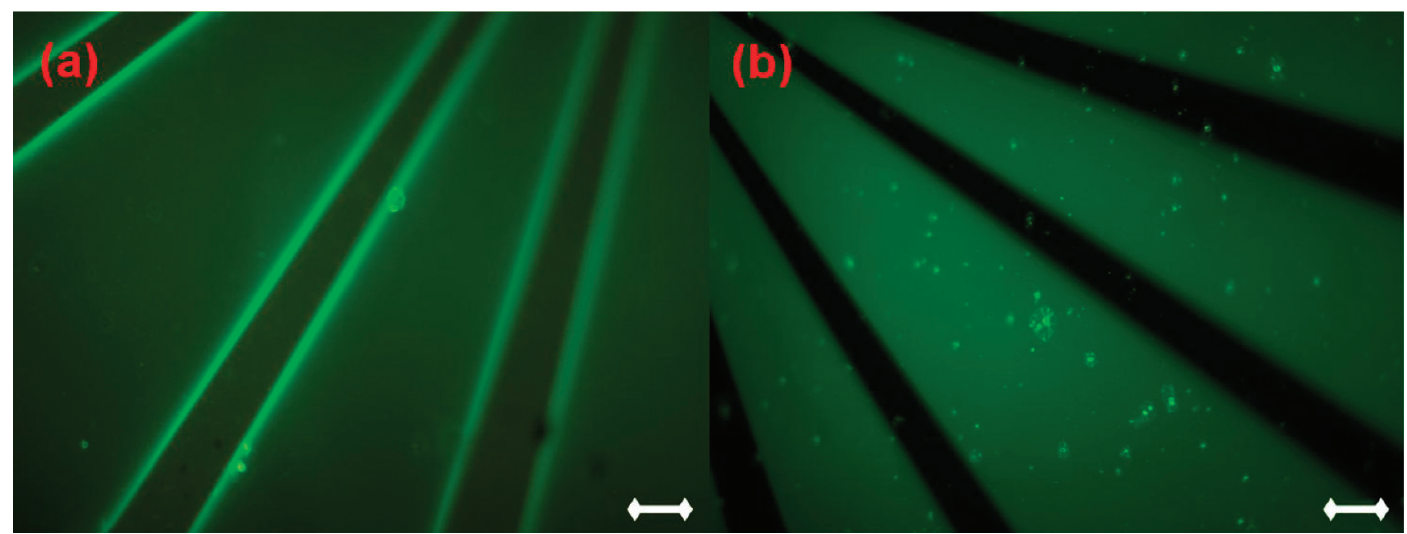

Figure 6. Fluorescence microscope images of PMMA with gold and R6G (a) and PMMA with only R6G (b) films, after photopatterning. The scale bar corresponds to $20 \mu \mathrm{m}$.

using the $6 \mathrm{~GB}$, we have observed that both gold nanoparticles and gold ions do quench its fluorescence but with different degrees; hence, it can serve as a contrast agent to differentiate between regions containing gold nanoparticles and ions.

PMMA films containing the Au salt and the R6G, and also PMMA films containing only the R6G, were prepared, and photopatterns on these films were imprinted with $24 \mathrm{~h} \mathrm{UV}$ irradiation. The fluorescence images of the resulting films were recorded with a fluorescence microscope. In the PMMA film with the gold and the R6G, regions with much brighter fluorescence than the rest have appeared at the borders between irradiated and masked regions as shown in Figure 6a. These regions are not observed in the PMMA film without gold (Figure $6 \mathrm{~b})$ and have comparable sizes to the postulated ion-depleted regions we have observed in our SEM images. As mentioned before, since both gold ions and also the gold nanoparticles quench the fluorescence of the R6G, these brighter regions enable the full fluorescence, since they are completely devoid of gold ions and nanoparticles. In order to exclude the possibility that the features we are observing might be due to photobleaching, we have also subjected the same patterning procedure to PMMA films without gold ions, where patterns are also observable but this time due to photobleaching. However, we should emphasize that we are interested in the fluorescence coming from the regions under the mask, which are not subjected to photobleaching; hence, our conclusions are valid, and the brighter features in the Figure 6a signal the absence of both of the gold moieties (ions and nanuparticles). In Figure 7, we superimposed the two fluorescence images in order to more clearly show that the ion-depleted regions are indeed located in the masked regions.

The evolution of ion-depleted regions with different irradiation doses can also be tracked using fluorescence images. Figure 8 shows the fluorescence images of the same film irradiated for $30 \mathrm{~min}, 1 \mathrm{~h}$, and $24 \mathrm{~h}$. It is seen that after $1 \mathrm{~h}$ the ion-depleted regions start to appear and the contrast gets sharper with increasing irradiation time. This gradual formation of the region may lead to important insight into the mechanism of $\mathrm{AuCl}_{4}{ }^{-}$ ion diffusion out of the dark regions toward the illuminated ones.

Another experimental evidence supporting the presence of these ion-depleted regions comes from X-ray photoelectron spectroscopy (XPS) mapping. XPS mapping/imaging is still not as precise as SEM imaging, and the quality of the images measured is limited to the spot size of the X-rays, which is 30 $\mu \mathrm{m}$ in our spectrometer. However, the chemical and surface specificity of XPS offsets some of this deficiency. The $30 \mu \mathrm{m}$ spot size is not small enough to realize an aerial scan, but a

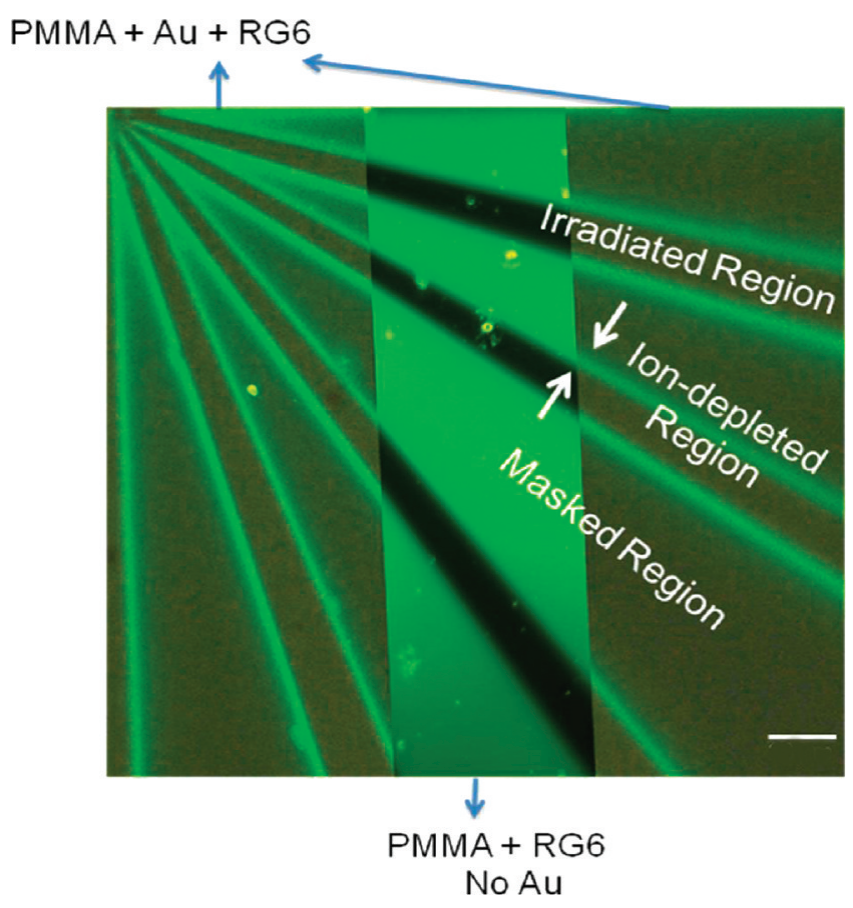

Figure 7. Fluorescence microscope images of PMMA with gold and R6G, the middle of which contains that of only R6G film superimposed. The scale bar corresponds to $20 \mu \mathrm{m}$.

line scan can give useful information. Two line scans of the Au4f spin-orbit doublet peak through the irradiated regions of our patterns from border to border are given in the insets of Figure 8 . The line scan was not taken from the masked regions since gold tends to move toward the surface during irradiation and thus can be detected better by XPS. As a result, we have performed the measurements on the irradiated regions, where we have expected to see more gold concentration near the borders, due to the diffused gold ions, compared to the middle irradiated regions. For the $24 \mathrm{~h}$ irradiated film, there was indeed more gold near the border regions, further proving that diffusion of gold ions from the dark regions toward the irradiated ones have taken place and formed ion-depleted regions of ca. $10 \mu \mathrm{m}$ length.

A similar observation was earlier reported by Sakamoto et al. ${ }^{20}$ for preparation of bimetallic $\mathrm{Au} / \mathrm{Cu}$ nanoparticles within PVAL matrix, where they observed formation of three distinct regions at the edges of their photopatterns with varying $\mathrm{Au} / \mathrm{Cu}$ ratios, resulting in particles with different sizes. They concluded that this was an effect of the transient metal intermediates' diffusion toward the edges, after ruling out a number of different 


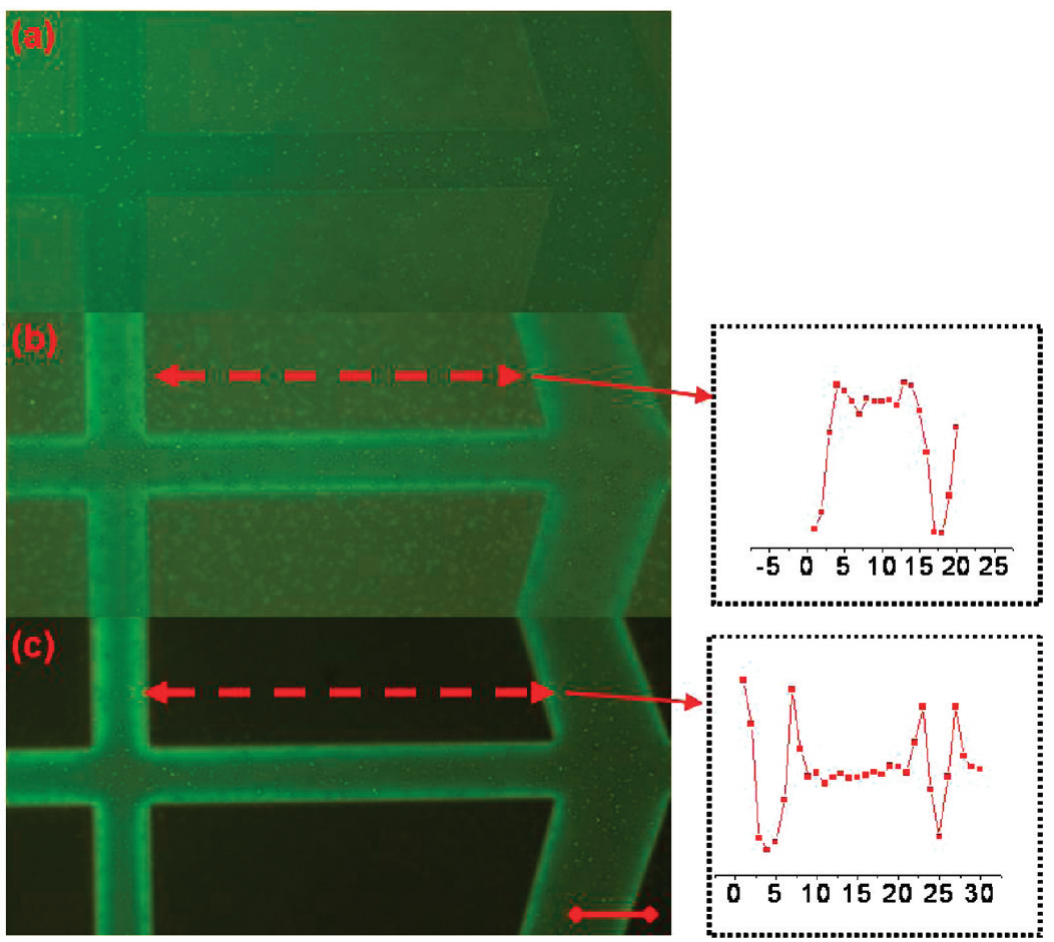

Figure 8. Fluorescence images of PMMA film with Au and R6G irradiated for (a) 30 min, (b) 1 h, and (c) 24 h. Inset: XPS line scans of the Au4f peak intensity. The scale bar corresponds to $50 \mu \mathrm{m}$.

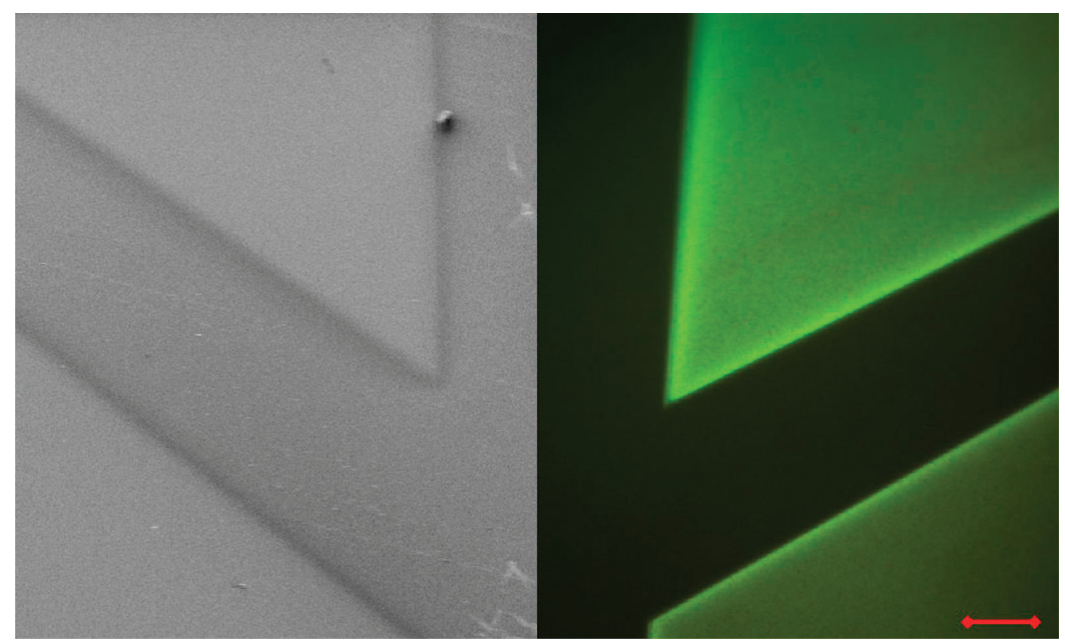

Figure 9. SEM (left) and fluorescence (right) images of photopatterned Au/PVAL films. The scale bar corresponds to $50 \mu \mathrm{m}$.

possible mechanisms to reach this conclusion. Diffusion of nanoparticles or electron donors, created by the action of the UV light on the carbon backbone of the polymeric matrix, are the other mentioned candidates, which were concluded to be invalid since nanoparticles cannot diffuse freely in polymer matrices and electron donors created have rather short lifetimes. To check that if we can also produce such ion-depleted regions in the PVAL matrix, we have prepared PVAL/Au films photopatterned like PMMA. We also observe the formation of ion-depleted regions at the borders (Figure 9), which shows that ion diffusion is not unique to PMMA.

It is well-known that gold atoms tend to come together, following their photoreduction step, which results in formation of gold nanoparticles, because of their large cohesive energy $(366 \mathrm{~kJ} / \mathrm{mol})$, but we are yet to know of a factor to force the gold ions to diffuse at a $10 \mu \mathrm{m}$ distance from the masked regions toward the irradiated ones at RT, and in the absence of external electrical potentials. Development of an electrochemical poten- tial due to depletion of ions as result of photoreduction in the irradiated regions can be thought of as the thermodynamic driving force, but its magnitude can be estimated as ca. 120 $\mathrm{mV}(11 \mathrm{~kJ} / \mathrm{mol})$ for an extreme concentration difference of a factor of 100 between the two regions. Even this value is about 30 times smaller than the driving force for the gold atoms.

Although somewhat scattered, there are several reports regarding the diffusion of gold atoms inside polymer matrices. Alexandrov et al. ${ }^{9}$ reported the diffusion coefficient $(D)$ of gold atoms in PMMA matrix at $348 \mathrm{~K}$ as $2 \times 10^{-7} \mathrm{~cm}^{2} \mathrm{~s}^{-1}$, which is quite large compared to other reports about diffusion of metal atoms in polymers. For example, in a detailed paper about diffusion of metal atoms in polymers by Faupel et al., ${ }^{10}$ the corresponding diffusion coefficients are given for gold atoms in different polymer matrices (like BPA-PC and TMC-PC) at temperatures about $500 \mathrm{~K}$. These reported values are in the range of $10^{-13}-10^{-16} \mathrm{~cm}^{2} \mathrm{~s}^{-1}$, smaller by about 7 orders of magnitude. In contrast to several studies on metal atoms, no corresponding 
reports are available dealing with diffusion of metal ions (i.e., $\mathrm{AuCl}_{4}{ }^{-}$) in polymer matrices. An approximate value can now be obtained by using our experimental results and the Fick's Law of diffusion

$$
s=-D / c(\partial c / \partial x)
$$

where $s$ denotes the terminal speed of the ions, $D$ is the diffusion coefficient, $c$ is the concentration, and $\partial c / \partial x$ is the concentration gradient. For a movement of $10 \mu \mathrm{m}=0.001 \mathrm{~cm}$ within $24 \mathrm{~h}$, we can get an estimate of $1.2 \times 10^{-8} \mathrm{~cm} / \mathrm{s}$ for $s$, and an upper limiting value for the concentration gradient can be estimated as $c / 10 \mu \mathrm{m}$, by assuming total consumption of the ions within the irradiated regions.

$$
D=1.2 \times 10^{-8} \mathrm{~cm} / \mathrm{s} * 0.001 \mathrm{~cm}
$$

This rough procedure yields an unreasonably large diffusion constant of $10^{-11} \mathrm{~cm}^{2} / \mathrm{s}$ for ions to move in polymer matrices at RT. Hence, further studies are definitely needed for understanding the processes involved for rational design and use of metal-polymer composites toward various applications.

\section{Conclusions}

Synthesis of gold nanoparticles in PMMA and PVAL matrices by UV irradiation presents a rather easy route for enabling photopatterning at room temperature. The photochemical process induces reduction of $\mathrm{Au}^{3+}$ ions and produces gold nanoparticles where light impinges. In addition, this process produces iondepleted regions of $10 \mu \mathrm{m}$ length, which sets an important and practical upper limit to the size of the lateral features for realization of durable and stable patterns. These regions form during irradiation of the films, where the gold ions from the masked regions are postulated to diffuse out toward the irradiated regions. Several imaging and spectroscopic techniques are used to characterize the size and the composition of these regions. The diffusion of $\mathrm{AuCl}_{4}{ }^{-}$ions within rigid polymer matrices like PMMA (or PVAL) for $10 \mu \mathrm{m}$ at room temperature is quite large and raises important questions about the driving force(s) behind this process.

Acknowledgment. We thank Prof. Kamil Can Akcali of Bilkent University and Prof. Dr. Mahinur Akkaya of Middle East Technical University for their kind help in obtaining fluorescence images. This work was partially supported by TUBA (Turkish Academy of Sciences) and by the European Union 7 Framework Project UNAM-REGPOT, Grant No. 203953.

\section{References and Notes} 18.

(1) Shipway, A. N.; Katz, E.; Willner, I. ChemPhysChem. 2000, 1, (2) Arango, A. C.; Carter, S. A.; Brock, P. J. Appl. Phys. Lett. 1999, $74,1698$.

(3) Balazs, A. C.; Emrick, T.; Russell, T. P. Science 2006, 314, 1107.

(4) Jiang, C. Y.; Markutsya, S.; Pikus, Y.; Tsukruk, V. V. Nat. Mater. 2004, 3, 721.

(5) Vitale, F.; Mirenghi, L.; Piscopiello, E.; Pellegrini, G.; Trave, E.; Mattei, G.; Fratoddi, I.; Russo, M. V.; Tapfer, L.; Mazzoldi, P. Mater. Sci. Eng., C 2007, 27, 1300.

(6) Horiuchi, S.; Fujita, T.; Hayakawa, T.; Nakao, Y. Adv. Mater. 2003, 15,1449 .

(7) Gorzolnik, B.; Mela, P.; Moeller, M. Nanotechnology 2006, 17, 5027.

(8) Korchev, A. S.; Bozack, M. J.; Slaten, B. L.; Mills, G. J. Am. Chem. Soc. 2004, 126, 10.

(9) Miyama, T.; Yonezawa, Y. J. Nanopart. Res. 2004, 6, 457.

(10) Korchev, A. S.; Konovalova, T.; Cammarata, V.; Kispert, L.; Slaten, B. L.; Mills, G. Langmuir 2006, 22, 375.

(11) Alexandrov, A.; Smirnova, L.; Yakimovich, N.; Sapogova, N.; Soustov, L.; Kirsanov, A.; Bityurin, N. Appl. Surf. Sci. 2005, 248, 181.

(12) Ozkaraoglu, E.; Tunc, I,; Suzer, S. Polymer 2009, 50, 462.

(13) Yilmaz, E.; Suzer, S. Appl. Surf. Sci. 2010, 256, 6630.

(14) Faupel, F.; Willecke, R.; Thran, A. Mater. Sci. Eng., $R$ 1998, 22,

(15) Sapogova, N.; Bityurin, N. Appl. Surf. Sci. 2009, 255, 9613.

(16) Dan, N.; Zubris, M.; Tannenbaum, R. Macromolecules 2005, 38, 9243.

(17) Kudelski, A.; Pettinger, B. Chem. Phys. Lett. 2000, 321, 356.

(18) Tanaka, T.; Yamaguchi, K.; Yamamoto, S. Opt. Commun. 2002, $212,45$.

(19) Sen, T.; Sadhu, S.; Patra, A. Appl. Phys. Lett. 2007, 91, 043104.

(20) Sakamoto, M.; Tachikawa, T.; Fujitsuka, M.; Majima, T. Adv. Funct. Mater. 2007, 17, 857.

JP106672F 